\title{
PROPUESTA DIDÁCTICA PARA EL USO DE PORTAFOLIOS DIGITALES EN EDUCACIÓN SUPERIOR
}

\section{DIDACTIC PROPOSAL FOR THE USE OF EPORTFOLIOS AT HIGHER EDUCATION}

\author{
Maria José Rubio Hurtado; mirubio@ub.edu \\ Cristina Galván Fernández; cgalvan@ub.edu \\ José Luis Rodríguez Illera; jlrodriguez@ub.edu
}

Universitat de Barcelona

\section{RESUMEN}

El artículo presenta una experiencia de innovación docente con portafolios digitales en ocho asignaturas de educación superior. Se ha utilizado la plataforma Carpeta Digital, la cual tiene funcionalidades que permiten optimizar el proceso de aprendizaje y el proceso de seguimiento por parte del profesorado. A través de los resultados satisfactorios en gran parte para estudiantes y profesorado, se propone una metodología de uso y recomendaciones para el trabajo con portafolios digitales.

PALABRAS CLAVE: Portafolio digital, educación superior, innovación, entornos de aprendizaje, guía docente.

\section{ABSTRACT}

This paper presents an experience of educational innovation with digital portfolios. The implementation has been done in eight subjects of higher education. We have used the platform Carpeta Digital, which has features that optimize the learning process, and the monitoring and feedback processes as well, by teachers. Thanks to successful results in a large sample of students and teachers, we propose an easy-to-use methodology and recommendations for working with digital portfolios.

KEYWORDS: E-portfolio, higher education, innovation, learning environments, teachers guides. 


\section{INTRODUCCIÓN}

La cuestión general de la evaluación y de las ayudas al aprendizaje se ha ido situando como algo central en muchas visiones de la docencia universitaria, especialmente en aquellas que buscan su renovación y adaptación al EEES. Pensada como evaluación alternativa, por la necesidad de mejorar tanto sus objetivos como su funcionamiento en relación a la más tradicional, o bajo la forma de evaluación continuada, por competencias, auténtica, formativa, o con otros nombres, la cuestión de la evaluación ha dejado de estar en un lugar de "lo ya sabido" para pasar a otro más problemático y complejo. Sin duda, muchos profesores universitarios consideran que se trata de algo estratégico pues las concepciones sobre la evaluación pueden conllevar cambios en las formas de su docencia y del aprendizaje de los estudiantes.

En nuestro caso, nos queremos centrar en el uso de una de esas herramientas de evaluación y aprendizaje que están siendo utilizadas de manera bastante general como son los sistemas de portafolios digitales. Los portafolios han sido ensalzados en numerosas ocasiones (Stefani, Mason y Pegler, 2007; Albuquerque y Laranjeiro, 2008; Buzzetto-More, 2010) como una manera, relativamente simple, de realizar un tipo de evaluación alternativa, más centrada en un enfoque de aprendizaje por evidencias, con posibilidades de ser ampliado a competencias, y un diálogo formativo entre el profesor y el estudiante (Rodríguez Illera, 2009; Cotterill et al., 2004; López Pastor, 2009 y Zubizarreta, 2004). En los últimos años, los portafolios digitales nos han acompañado en procesos de innovación docente y de investigación desde el diseño y desarrollo de una plataforma denominada Carpeta Digital.

En este sentido, el artículo presenta una propuesta de innovación educativa centrada en la evaluación de los estudiantes universitarios a través de la plataforma Carpeta Digital, así como un estudio exploratorio de su funcionamiento desde la perspectiva de estudiantes y docentes implicados en la innovación. Presentaremos primero este sistema de portafolios, en segundo lugar los pormenores de cómo es utilizado en la práctica cotidiana de nuestra enseñanza universitaria para terminar analizando la opinión de profesores y estudiantes y proponiendo una Guía de uso docente de Carpeta Digital .

\section{LA PLATAFORMA CARPETA DIGITAL COMO SISTEMA DE PORTAFOLIOS}

La elección de la plataforma Carpeta Digital ha tenido en cuenta un análisis previo de los sistemas de portafolios existentes (López Fernández y Rodríguez Illera, 2009) y ha privilegiado las funcionalidades específicas de Carpeta Digital frente a las que presentan otras plataformas (Rodríguez Illera, 2009). Funcionalidades que permiten tanto una mayor sostenibilidad y optimización del proceso de seguimiento del aprendizaje (herramientas de calificación, diálogo con cada estudiante sobre las evidencias y visualización del desarrollo de las competencias), como una mayor optimización del proceso de aprendizaje (autoevaluación de competencias, multi-portafolios, diálogo con el docente sobre las evidencias y permanencia de la plataforma, entre otros). 


\section{Las funcionalidades de Carpeta Digital}

El conjunto de funcionalidades dependen del tipo de usuario o rol dentro del sistema (estudiante, profesor y administrador) y se concretan en:

a. La gestión documental y construcción de portafolios: Los estudiantes tienen disponible un espacio personal y privado en el que pueden guardar y crear documentos nuevos para (1) etiquetarlos con las competencias que se evidencian en los mismos y (2) organizarlos y visualizarlos en portafolios digitales.

b. El intercambio e integración de los documentos y portafolios: El portafolios se puede exportar en un archivo descargable con los contenidos del mismo y con un formato html para poder verlo de nuevo como una página web. De esta manera se puede visualizar el portafolios off-line.

c. La visualización y publicación de los resultados: El portafolios es privado del estudiante hasta el momento en que lo envía al profesor. De este modo sólo lo ven el estudiante y el profesor en formato de página web. También se puede publicar los portafolios en la red creando una dirección web para poder presentarlos en cualquier otro contexto académico, profesional o personal.

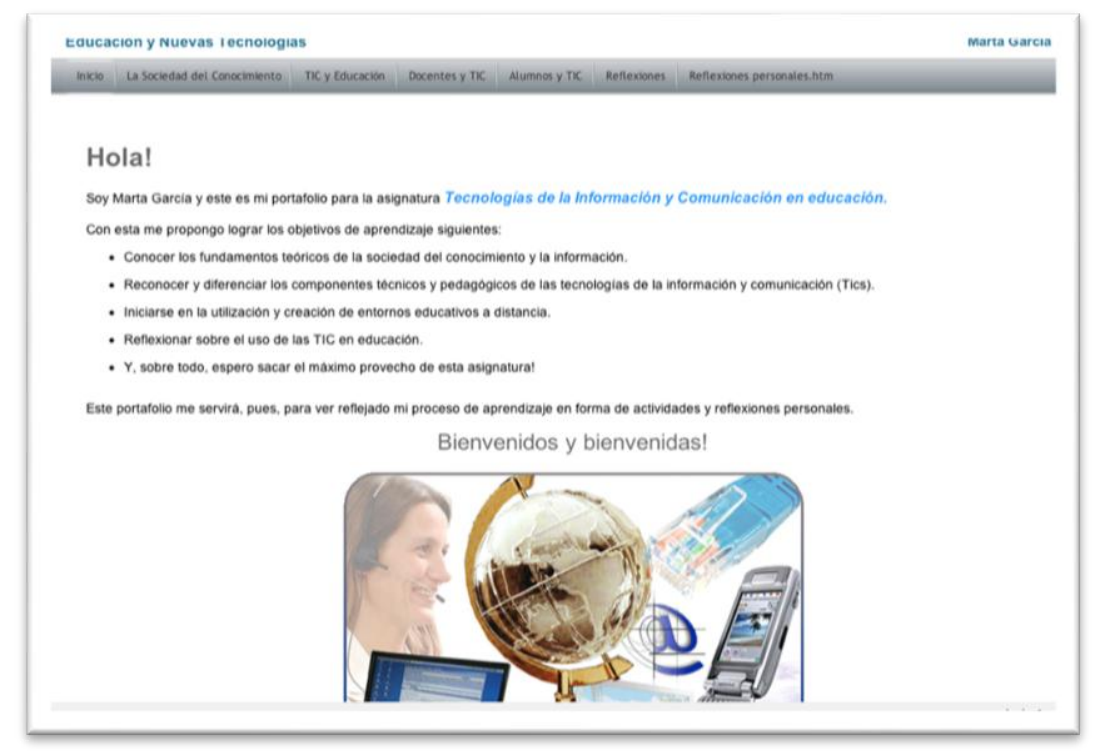

Imagen 1. Vista de un portafolios académico en el que toda la información está en formato html para facilitar la navegación.

d. La interacción y comunicación entre los usuarios del sistema: Los profesores y los estudiantes pueden realizar el seguimiento de los aprendizajes mediante mensajes textuales e indicaciones del estado del seguimiento así como mediante la asignación de calificaciones cualitativas o cuantitativas. La pantalla principal ofrece una visualización previa y un acceso directo a la pantalla de Diálogo, desde la que se gestiona esta funcionalidad. 


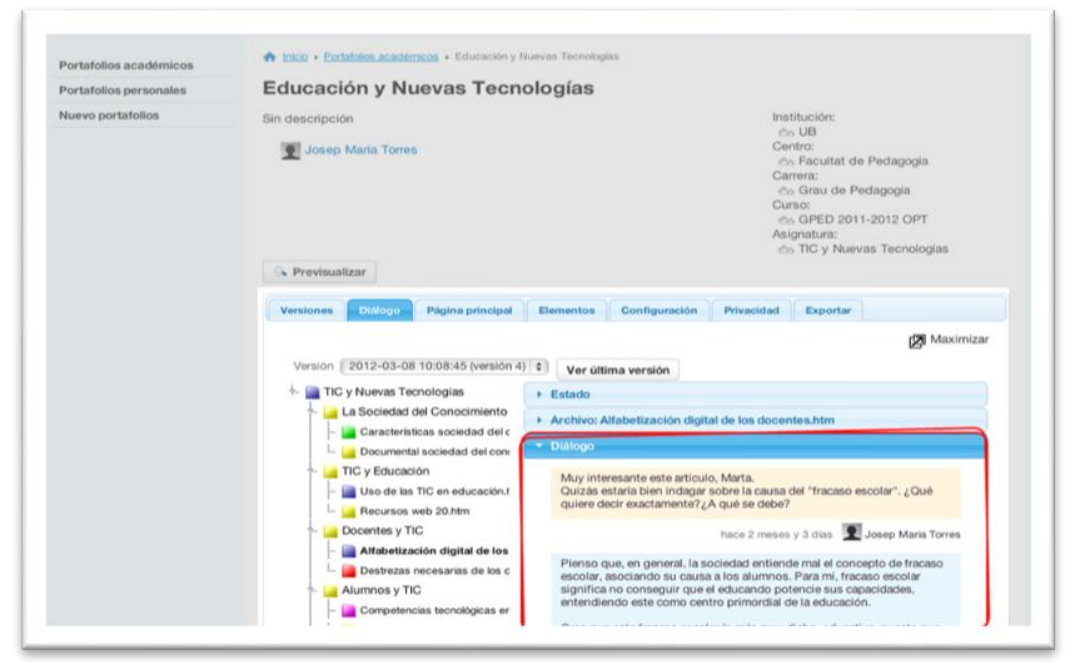

Imagen 2. Diálogo entre docente y estudiante sobre un documento o evidencia del portafolios.

e. La gestión del seguimiento de las competencias y de la evaluación del portafolios: Los estudiantes pueden etiquetar las evidencias de aprendizaje con las competencias (definidas por la institución o bien personales) que están desarrollando y auto-evaluar el grado de progreso de cada competencia. Así el estudiante puede conocer con qué tipo de documentos las está demostrando y detectar necesidades formativas.

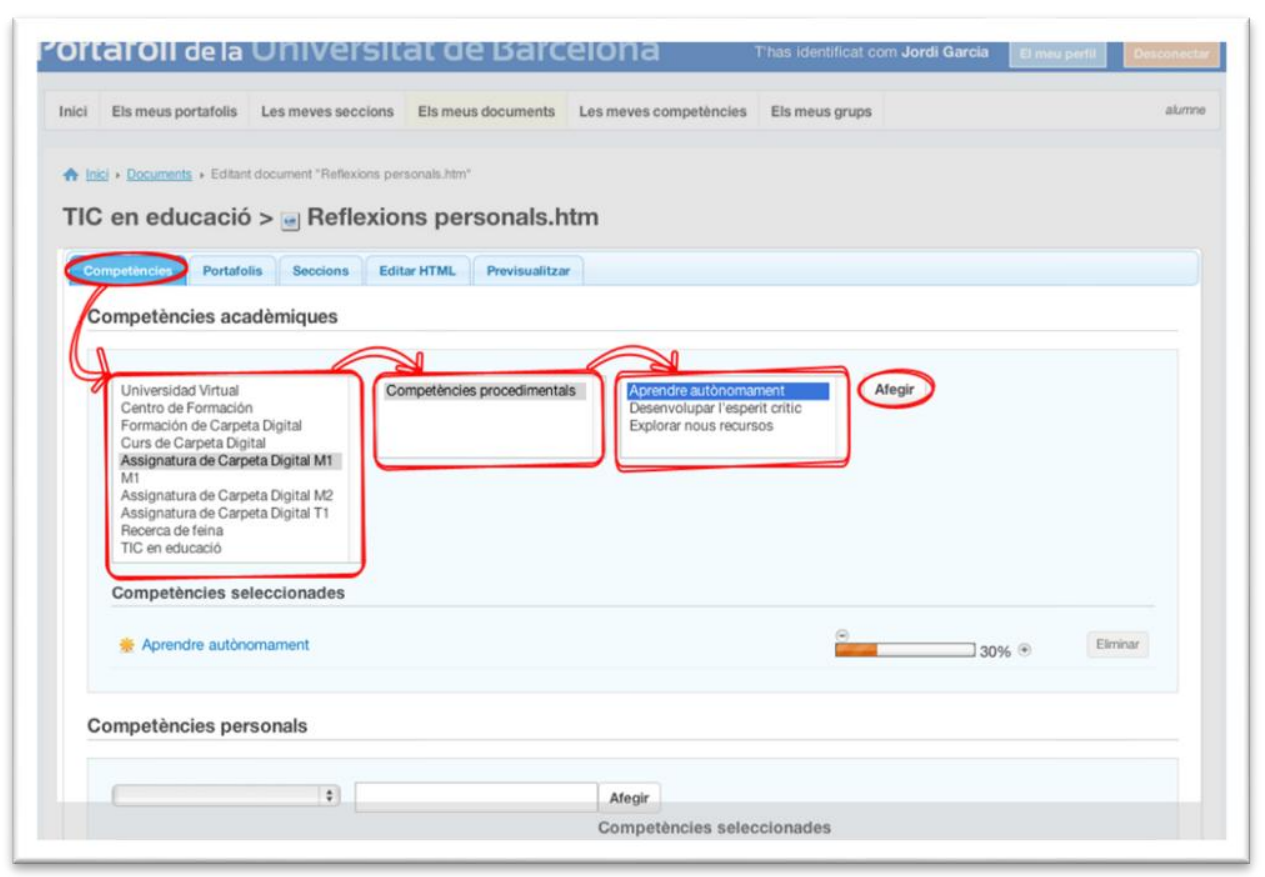

Imagen 3. Selección de competencias asociadas a un documento y que pertenecen a las definidas desde una comunidad (institución, facultad, titulación o asignatura).

Por otro lado, el profesor cuenta con funcionalidades que facilitan la tarea de evaluación, tales como reconocer qué portafolios ha visualizado o ha comentado, si tienen alguna respuesta pendiente o qué calificaciones ha asignado. 
f. La gestión por comunidades de aprendizaje: el sistema permite asignar portafolios y competencias a los estudiantes en función del contexto formativo: institución (universidad), facultad, titulación, asignatura y/o grupos de trabajo. Por ejemplo, un estudiante tendrá acceso a aquellos portafolios y competencias que le hayan sido asignados en función de las comunidades a las que pertenezca.

Cada comunidad se puede gestionar individualmente o desde niveles superiores. Es decir, un administrador de facultad podrá dar de alta a los usuarios de su facultad y asociarlos a los portafolios concretos así como determinar qué competencias (definidas desde la facultad) deben asociarse a los aprendizajes. Un profesor, por su parte, tiene permiso para asociar las competencias que son de su asignatura si es también administrador de la misma.

\section{UNA PROPUESTA DE INNOVACIÓN EDUCATIVA}

La propuesta de innovación ha consistido en el uso de Carpeta Digital, y su metodología didáctica asociada, en asignaturas de diferentes estudios de la Universidad de Barcelona. Estos han sido:

1. Estudios de Pedagogía y Maestro: Pedagogía de la Comunicación, Pedagogía de la Formación a Distancia, Educación Moral y Nuevas tecnologías aplicadas a la Educación (Maestro).

2. Estudios de Comunicación Audiovisual: Investigación de Medios en Educación y Multimedia y telemática Educativa.

3. Estudios de Derecho: Prácticum III y Derecho Mercantil.

4. Máster Enseñanza y Aprendizaje en Entornos Digitales: Consecuencias Pedagógicas de la Sociedad de la Información y Diseño Pedagógico aplicado a la Educación.

El profesorado que imparte estas asignaturas comparte una experiencia previa en el uso de portafolios digitales y también en portafolios en papel. $Y$ entre sus objetivos docentes figuran: la mejora del proceso de seguimiento de los aprendizajes de los estudiantes según orientaciones del EEES (Espacio Europeo de Educación Superior), y el hacer uso de entornos virtuales de aprendizaje y de nuevas metodologías docentes (GID-EAV, 2012).

Para la implementación de Carpeta Digital en las asignaturas mencionadas el profesorado consensuó una metodología de uso teniendo en cuenta: a) la preparación del proceso de enseñanza-aprendizaje, b) la formación sobre el portafolios y uso de la plataforma, c) las actividades a desarrollar durante el curso, y d) el seguimiento y evaluación del proceso de aprendizaje. Esta metodología didáctica se ha plasmado en una Guía Didáctica, que sirve de orientación a los docentes que utilizan la Carpeta Digital.

\section{a. Preparación del proceso de enseñanza-aprendizaje}

Es importante que los estudiantes comprendan desde un primer momento qué implicaciones va a tener el seguimiento continuo de los aprendizajes. Por ello en la sesión de presentación del plan docente se les debe ofrece una explicación breve de lo que son los 
portafolios digitales, qué se pretende conseguir, qué les va a aportar y cómo los van a realizar. También debe destacarse el proceso formativo de realización, los contenidos y el sistema de evaluación.

\section{b. Formación sobre el portafolios y uso de la plataforma}

La mayoría de los estudiantes suelen poseer experiencia con portafolios en papel, en los que han seguido una metodología centrada en: una colección de apuntes y actividades y una auto-evaluación entregada al finalizar el curso. Por ello el paso a un portafolios digital no debe suponer un problema importante, pero si es necesaria una formación relacionada con la formación pedagógica sobre portafolios digitales y sobre la plataforma.

El objetivo principal de esta formación es que los estudiantes consideren el portafolios digital como un producto propio, que debe reflejar todo el aprendizaje adquirido durante una asignatura, vida académica o vida profesional. La representación del aprendizaje adquirido puede verse mejorada en un entorno digital gracias al uso de distintos medios, como los que pueden ser utilizados en un portafolios multimedia, que contribuye a visualizar mejor los logros y procesos realizados (Barberà, 2009).

La formación se complementa con la muestra de buenas prácticas de otros estudiantes, consistentes en portafolios que están bien organizados según el tipo de actividad. Por último, se presentan otros usos -no académicos- para fomentar la motivación en la realización del portafolios y su continuación fuera del contexto universitario.

La formación sobre la plataforma contempla también aspectos formales como la estructura de los documentos, la organización en secciones y la creación y edición de archivos.

\section{c. Durante el curso: actividades de aprendizaje}

Los portafolios nos muestran las actividades de aprendizaje que el estudiante ha realizado a lo largo del proceso formativo (Jafari y Kaufman, 2006). Estas actividades pueden ser obligatorias, voluntarias o personales y no tienen por qué ser de la misma tipología. Los docentes deben optar por solicitar y/o recomendar aquellas que mejor se adaptaban a los objetivos y/o competencias a alcanzar en sus asignaturas.

Se considera interesante que el estudiante sea capaz de organizar las evidencias de aprendizaje mediante algún criterio académico con la finalidad de: i) lograr coherencia entre los portafolios de todas las asignaturas, y ii) orientar al estudiante que no tiene experiencia en la organización de evidencias. Estas formas organizativas de las evidencias (denominadas secciones en terminología de Carpeta Digital) establecidas de forma común son:

i) Actividades de aula u obligatorias: Asociación de los documentos o evidencias de aprendizaje correspondientes a actividades que se realizan en el aula o que son obligatorias en la asignatura.

ii) Proyecto (en caso de haberlo): Se incluyen los documentos correspondientes al proyecto. En general: la propuesta del proyecto, la propuesta mejorada del proyecto y la justificación de los cambios, descripción de las acciones realizadas y pendientes 
del diseño del proyecto (dificultades y resolución de problemas) y diseño final del proyecto.

iii) Actividades complementarias: Documentos que se refieren a actividades realizadas por el estudiante de manera voluntaria o que están en el marco de otras asignaturas, pero relacionadas con la asignatura de manera justificada. Sirven para complementar la visión del aprendizaje adquirido que se está mostrando en el portafolios y para transmitir la capacidad de construcción de la globalidad curricular, así como la comprensión de la situación de una asignatura dentro de una red de conocimientos (como es la titulación o un tema de interés).

iv) Reflexiones: Incorporadas dentro de los documentos asociados a las anteriores secciones y que, implícita o explícitamente, muestran las evidencias de aprendizaje. El objetivo de esta sección es incorporar las reflexiones que surgen de la realización de actividades, de las sesiones presenciales, de las lecturas, etcétera, y que pueden responder a preguntas tales como: ¿qué he aprendido?, ¿cómo transfiero estos conocimientos a otros campos o a una futura práctica profesional?, ¿qué ha dificultado mi aprendizaje?,... (BECTA, 2007; Dysthe y Engelsen, 2004).

Además de las anteriores, los estudiantes pueden añadir otras secciones a su portafolios con otro tipo de documentos o incluso tener un portafolios con secciones de su preferencia en vez de las recomendadas por el profesorado.

\section{d. Seguimiento y evaluación del proceso de aprendizaje}

La evaluación continua y pautada, incluye:

i) La retro-alimentación a través de la función Diálogo de la plataforma en una de las dos modalidades: (a) mediante mensajes sobre el contenido de un documento, de una sección o del portafolios en general, siendo contestados por el estudiante; (b) mediante la identificación de un estado de revisión del portafolios (iniciado, apto, no apto, con un diálogo finalizado).

ii) La revisión de los portafolios durante un periodo de dos semanas desde la última fecha de entrega establecida, y centrada tanto en aspectos formales de presentación como de contenidos de la materia y de reflexión.

iii) La utilización de un sistema de rúbrica para evaluar distintos componentes del portafolios, rúbrica que se presenta al alumnado al comienzo de la asignatura, ejerciendo así una función de evaluación formativa.

La metodología de uso del portafolios incluye la propuesta de una planificación en las fechas de entrega de las evidencias de aprendizaje y una evaluación y retro-alimentación de las mismas dentro de la propia Carpeta Digital.

\section{ESTUDIO DE LA INNOVACIÓN EDUCATIVA}

Para comprobar cómo había funcionado la implementación de Carpeta Digital desde la perspectiva de los usuarios, se recogió información tanto de los estudiantes como de los 
docentes. La finalidad fue conocer la viabilidad de la innovación desde el punto de vista de los profesores a partir de las consecuencias de su uso en la organización de la docencia; y conocer cómo los estudiantes perciben el uso de los portafolios y de la plataforma en el proceso de aprendizaje.

La información se obtuvo a través de las siguientes estrategias (tabla 1):

- Una entrevista individual dirigida a los 5 profesores implicados en la implementación para conocer la valoración de la innovación. Los cinco profesores imparten las 8 asignaturas mencionadas anteriormente (habiendo profesores que imparten más de una asignatura).

- Un cuestionario semi-estructurado on-line dirigido a todos los estudiantes del total de asignaturas implicadas, formado por preguntas cerradas (definidas como una escala Likert ) y por preguntas abiertas, con tal de complementar o justificar las respuestas de las preguntas cerradas. El cuestionario se respondía de forma voluntaria.

\begin{tabular}{|c|c|c|}
\hline Instrumento & Informantes & Información \\
\hline Entrevista & $\begin{array}{l}\text { Profesorado } \\
\text { implicado }(\mathrm{N}=5)\end{array}$ & $\begin{array}{l}\text { - Funcionamiento de Carpeta Digital (CD) } \\
\text { - Cambios percibidos en la metodología docente } \\
\text { - Valoración de CD }\end{array}$ \\
\hline Cuestionario & $\begin{array}{l}\text { Estudiantes } \\
\text { implicados ( } \mathrm{N}=150)\end{array}$ & $\begin{array}{l}\text { - Uso de CD } \\
\text { - Utilidad y adecuación de CD } \\
\text { - Valoración de CD }\end{array}$ \\
\hline
\end{tabular}

Tabla 1. Estrategias de obtención de información

Los resultados principales del seguimiento de la innovación se presentan separados según los informantes.

\section{a) La perspectiva del profesorado respecto la metodología por portafolios y la plataforma}

La entrevista fue contestada por los cinco docentes y confirmó el uso de una metodología común propuesta por parte de todo el profesorado implicado, tal y como se ha descrito en el apartado anterior. En concreto se evidenciaron los siguientes aspectos:

i) Planificación de la asignatura

El profesorado integró el sistema de portafolios en la planificación de sus asignaturas, proponiendo una evaluación continua con diferentes entregas a lo largo del curso, aspecto considerado como necesario por el propio profesorado a fin de favorecer el seguimiento del aprendizaje de los estudiantes en todo momento. Las actividades propuestas y el número variaron de unas asignaturas a otras, pero el aspecto común fue plantear un porcentaje elevado dentro de la obligatoriedad y un porcentaje mínimo dentro de la optatividad (una sola actividad de libre elección, pero de obligada presentación).

ii) Valoración de la plataforma

La valoración de los profesores fue positiva, estableciendo como argumento principal la buena gestión de la evaluación a través de las evidencias organizadas en secciones y de la 
funcionalidad del Diálogo que integra Carpeta Digital, aspecto que facilita la comunicación con el estudiantado, favoreciendo la tarea docente.

iii) Valoración de los portafolios

La valoración de los portafolios se estableció según una rúbrica de diferentes dimensiones como el aspecto organizativo, de contenido y de diseño, lo que favoreció la objetividad a la hora de valorar los portafolios. Los profesores constataron una mejora progresiva de los portafolios a medida que la plataforma y la didáctica se integraban en la metodología de trabajo de los estudiantes, destacando la idea de que deben habituarse a trabajar con el portafolios digital para entregar un producto de calidad. Por ello recomiendan el uso del portafolios también en sesiones presenciales, a fin de que los estudiantes tomen conciencia y lo trabajen de manera continuada.

iv) Percepción sobre los estudiantes

Los profesores destacaron que sus estudiantes se sentían satisfechos una vez acabado el portafolios. El esfuerzo dedicado merece la pena por el producto final conseguido y la resistencia inicial mostrada por algunos estudiantes hacia una nueva plataforma desaparece una vez se han habituado a su uso.

v) Viabilidad de la innovación

El profesorado admitió un aumento de trabajo en su tarea docente, pero asumible especialmente con grupos no excesivamente numerosos. La revisión continuada y mediante rúbrica de los portafolios y una rápida retro-alimentación sobre las evidencias de aprendizaje son los inconvenientes principales que proponen los docentes con grupos numerosos y en un contexto de evaluación continuada.

\section{b) La perspectiva de los estudiantes}

Del total de 150 estudiantes, 43 contestaron el cuestionario, destacando los siguientes aspectos en sus respuestas:

i) Usos destinados de la plataforma

El $100 \%$ de los estudiantes utilizaron la plataforma únicamente para colgar y publicar las actividades de la asignatura, mientras que un 2,9\% también la usó para guardar otros documentos académicos que no eran de alguna asignatura y un 5,7\% para guardar documentos personales no académicos.

ii) Valoración del hecho de tener un espacio privado y editable on-line

La valoración de los estudiantes en referencia al hecho de tener un espacio privado en línea donde almacenar información fue muy positiva, puesto que un 37,14\% lo consideró muy interesante, un $40 \%$ lo consideró bastante interesante, un $20 \%$ interesante y sólo un 2,86\% de los estudiantes que respondieron al cuestionario lo consideró poco interesante. 
iii) Utilidad de la plataforma

Según los estudiantes, Carpeta Digital resultó útil en los siguientes aspectos:

- Principalmente para la entrega de actividades (con una media de 4,17 en una escala Likert de cinco puntos) y la re-edición de las actividades (con una media obtenida de 3,97 ).

- También la consideraron útil por mantener la privacidad en el desarrollo de las actividades (con una media de 3,85 ) y para el seguimiento de la asignatura (con una media de 3,57).

- En cambio por el hecho de un uso personal y no únicamente académico de la herramienta no la consideraron tan interesante (con una media de 2,65 ).

iv) Ventajas y/o inconvenientes de Carpeta Digital

Las ventajas e inconvenientes considerados por los estudiantes respecto a Carpeta Digital quedan reflejados en la tabla 2.

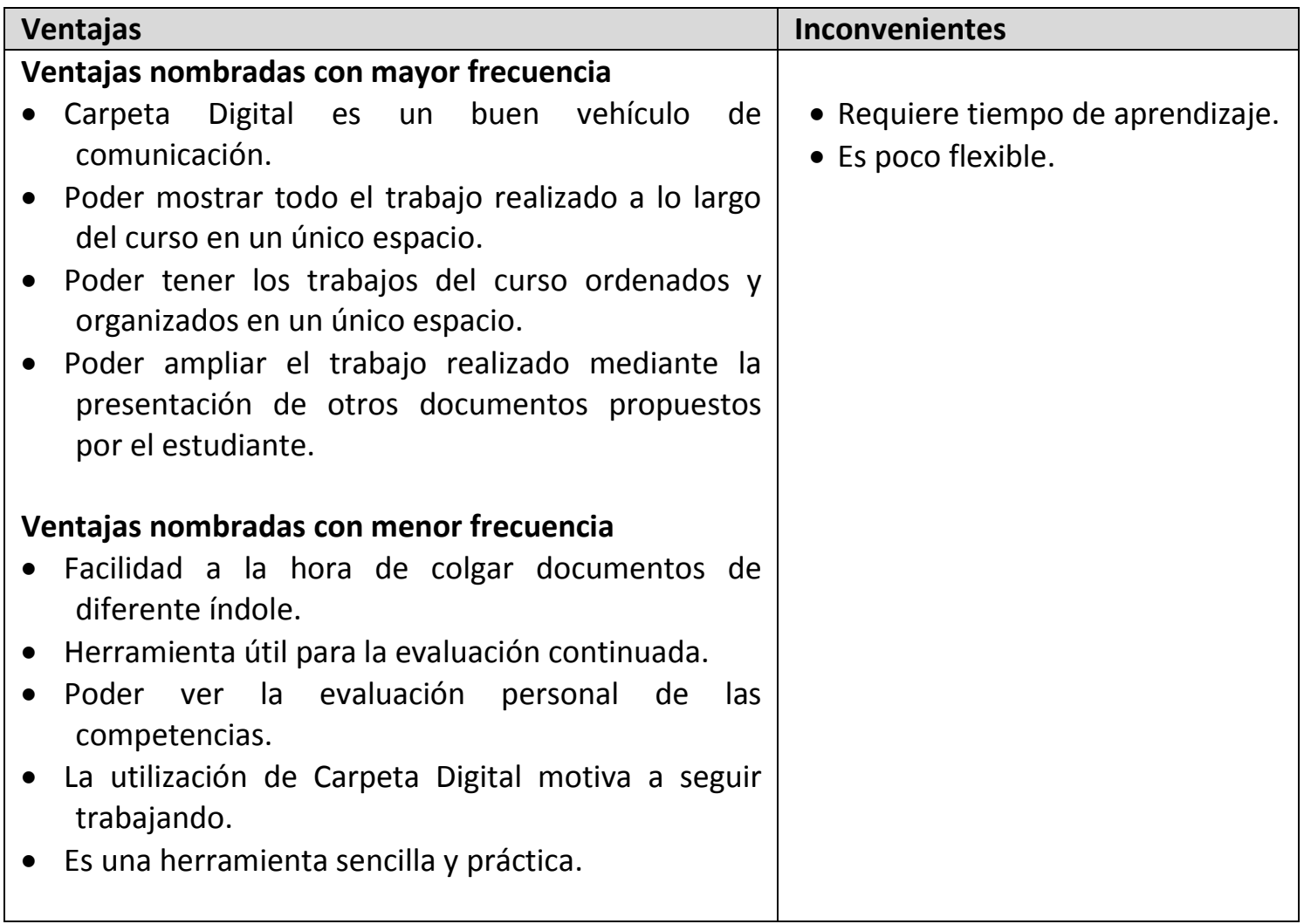

Tabla 2. Ventajas e inconvenientes de Carpeta Digital manifestadas por los estudiantes

\section{DISCUSIÓN Y PROSPECTIVA}

\section{Discusión}

Los datos nos permiten afirmar que la aplicación diseñada es una herramienta útil para los usuarios y con un interesante potencial pedagógico, para las necesidades emergentes, como es el proceso de enseñanza-aprendizaje por competencias. 
Desde la perspectiva del profesorado que la ha utilizado, éste la percibe como un instrumento que no sólo le ayuda a gestionar la evaluación de sus estudiantes, sino que impacta directamente en la planificación de su asignatura, proporcionándole un marco para repensar, sistematizar y mejorar su práctica evaluativa dirigiéndola a que los alumnos evidencien sus aprendizajes a través de actividades tanto pautadas por el docente como libremente escogidas por el estudiante. Aunque la ratio del alumnado es un tema sobre el que cabe reflexionar para ofrecer una metodología didáctica que permita una gestión sostenible con grupos numerosos también. El sistema de rúbricas para la valoración del portafolios contribuye tanto a un aumento de la dedicación del profesorado como a una mayor objetividad de la valoración.

En relación al alumnado, éste valora la metodología de uso propuesta, en cuanto al propio seguimiento del aprendizaje y por la posibilidad que ofrece de una autoevaluación continuada. La percepción que los estudiantes tienen de Carpeta Digital es la de una herramienta interesante en su conjunto, considerando que les ayuda a hacer un buen seguimiento de los contenidos y a reflexionar sobre su propio aprendizaje.

Los usos más personales de la herramienta y la explotación de todas sus funcionalidades son aspectos menos integrados por el alumnado, aspecto que podría mejorar con un uso más a largo plazo y que habrá que comprobar en proyectos futuros. El uso a largo plazo permite la familiarización de la herramienta durante un primer curso y la optimización de su potencial pedagógico durante el segundo y los siguientes.

Una vez se ha iniciado el curso, los estudiantes no suelen estar acostumbrados a este tipo de prácticas por lo que hay que dedicar una parte del tiempo lectivo al trabajo con portafolios, así como orientar de forma más directa a los estudiantes en la creación de las evidencias y de los portafolios. En este último aspecto entran en juego el papel técnico y el pedagógico. Aunque es de esperar que cada vez menos sea necesario realizar sesiones de funcionamiento de la herramienta, por la incorporación de manuales de uso y por la propia alfabetización digital de los estudiantes.

\section{Prospectiva}

La experiencia con la herramienta genera también nuevas ideas, tanto técnicas como pedagógicas, tanto de diseño como de utilización, que contribuirán a afianzar el ciclo de diseño-implementación, y a mejorar tanto el sistema como su adecuación a las situaciones reales de enseñanza y aprendizaje.

Expandir la experiencia a otros ámbitos, estudios y docentes, facilitando la incorporación a través de una guía didáctica que ofrece pautas al profesorado sobre el tratamiento didáctico con Carpeta Digital será un objetivo a medio plazo a fin de validar la adecuación de la herramienta en diferentes contextos.

Para potenciar el desarrollo de portafolios más complejos, igual que para fomentar el uso personal de la herramienta se apostará por la posibilidad de que los estudiantes recuperen esta metodología en varias asignaturas durante períodos continuados, incluso que se integre en un ciclo que abarque el conjunto de los años de carrera. Se considera importante conseguir que los estudiantes integren la herramienta en su proyecto formativo y profesional, a fin de que la utilidad del portafolios se extienda más allá del propio periodo educativo. 
Los portafolios tienen el potencial para ser un tipo privilegiado de Entorno personal de aprendizaje (PLE), es decir una herramienta consolidada de asistentes personales de gestión y planificación del aprendizaje, así como de desarrollo personal, en múltiples contextos. Y en este sentido, en futuras versiones de Carpeta Digital se prevé la incorporación en su diseño de un entorno de estas características.

\section{CONCLUSIONES}

El objetivo perseguido de implementar una experiencia de evaluación con un sistema de portafolios digital (Carpeta Digital) y valorar dicha experiencia desde el punto de vista de sus usuarios se ha cumplido. Ello ha permitido optimizar la toma de decisiones en relación a la continuación en el uso de la herramienta.

Por un lado, la valoración positiva de la experiencia en conjunto por parte de los profesores ha dado pie a su continuación durante el curso siguiente con Carpeta Digital por parte del mismo grupo de docentes, así como por otros nuevos que han incorporado el uso de esta plataforma y el tratamiento didáctico propuesto. Aunque éste deberá acabar de ajustarse para poder adaptarse a grupos de alumnado de diferentes tamaños.

Por otro lado, hemos comprobado como los estudiantes van aceptando la metodología de uso del portafolios y encontrando ventajas en el uso académico del sistema Carpeta Digital, lo cual es una fuente de motivación para seguir usando la herramienta. Los resultados obtenidos en la experiencia desde la perspectiva de los estudiantes corresponden a un uso novel durante un semestre, por lo que será interesante conocer cuáles son los resultados en un uso a medio o largo plazo. De este modo se verá si mejoran las ventajas y/o disminuyen los inconvenientes.

\section{AGRADECIMIENTOS}

Los autores agradecen la financiación recibida del Ministerio de Ciencia e Innovación a través del proyecto EDU2008-01458 y del Ministerio de Economía y Competitividad a través del proyecto EDU2012- 37897

\section{BIBLIOGRAFÍA}

ALBUQUERQUE, F. Y LARANJEIRO, M. (2008). E-portfolio in Education. Practices and Reflections. Coimbra: Associação de Professores de Sintra.

BARBERÀ, E. y Martín, E. (2009). Portfolio electrónico: aprender a evaluar el aprendizaje. Barcelona: UOC.

BECTA (2007). Impact study of e-portfolios on learning. En línea: http://partners.becta.org.uk/index.php?section=rh\&catcode= re rp 02\&rid=14007 [Consultado: 26/06/2008]

BUZZETTO-MORE, N. (2010.) The Portfolio Paradigm: Informing, Educating, Assessing, and Managing With E-Portfolios. California: Informing Science Press. 
COTTERILL, S.; MCDONALD, T.; DRUMMOND, P. Y HAMMOND, G. (2004). Design, Implementation and Evaluation of a "generic" ePortfolio: The Newcastle Experience. ePortfolio 2004 Conference. La Rochelle, 28-29 octubre.

DYSTHE, O., \& ENGELSEN, K. (2004). Portfolios and assessment in teacher education in Norway: A theory-based discussion of different models in two sites. Assessment \& Evaluation in Higher Education, 29(2), 239-258.

GARRISON, D. Y CLEVELAND-INNES, M. (2005). Facilitating Cognitive Presence in Online Learning: Interaction Is Not Enough. American Journal of Distance Education , 19(3), 133-148

Grupo de Innovación Docente -Enseñanza y Aprendizaje Virtual (2012). Objetivos GID-EAV. En línea: http://greav.ub.edu/grupo id EAV/objetivos gid eav.html [Consultado: 26/06/2012]

JAFARI, A. Y KAUFMAN, C. (eds, 2006). Handbook of Research on ePortfolios. Hershey (PA): Idea Group.

LÓPEZ FERNÁNDEZ, O., RODRÍGUEZ ILLERA, J.L. (2009). Investigating university students'adaptation to a digital learner course portfolio. Computers \& Education, 52 (3), 608-616.

LÓPEZ PASTOR, V. (2009). Evaluación Formativa y Compartida en Educación Superior. Propuestas, técnicas, instrumentos y experiencias. Madrid: Narcea.

RODRÍGUEZ ILLERA, J.L. (2009). Los portafolios digitales como herramientas de evaluación y de planificación personal. En: M. Castelló (comp). La evaluación auténtica en Enseñanza Secundaria y universitaria: investigación e innovación. Barcelona: Edebé.

RODRÍGUEZ ILLERA, J.L., RUBIO, M.J., GALVÁN, C., CAMPOS, E. Y AIXENDIR, R. (en prensa). L'ús dels portafolis digitals per l'avaluació per competències i continuada dins de I'EEES. RECERCAT Dipòsit de la Recerca de Catalunya [publicación online].

STEFANI, L., \& MASON, R., \& PEGLER, C. (2007). The educational potential of e-portfolios. Routledge: London and New York.

STEVENS, D. I LEVI, A. (2005) Introduction to rubrics. Virgina: Stylus.

ZUBIZARRETA, J. (2004). The learning portfolio. San Francisco: Anker.

\section{Para citar este artículo:}

RUBIO, M.J., GALVÁN, C. \& RODRÍGUEZ J.L. (2013). Propuesta didáctica para el uso de portafolios digitales en educación superior. EDUTEC, Revista Electrónica de Tecnología Educativa, 43. Recuperado el dd/mm/aa de http://edutec.rediris.es/Revelec2/Revelec43/propuesta didactica portafolios digitales educacion $\mathrm{s}$ uperior.html 